\title{
Un modelo para educar y evaluar habilidades de comunicación en estudiantes de medicina
}

\section{A model to educate and evaluate communicative abilties of medical students}

\author{
Pedro Esteves ${ }^{1}$, Ignacio Torres ${ }^{1}$, Alejandra Mampel ${ }^{1}$, Iván Fernández ${ }^{1}$, Marta Marianetti ${ }^{1}$. \\ 1Universidad Nacional de Cuyo. Facultad de Ciencias Médicas. Mendoza. Argentina. \\ Correspondencia: Pedro Eliseo Esteves; e-mail: pedroeesteves@gmail.com.
}

\section{Resumen}

Las habilidades de comunicación constituyen una competencia fundamental en profesionales de la salud. Educarlas fomenta el profesionalismo en estudiantes y graduados. El objetivo del presente trabajo es describir un curso diseñado para optimizar la comunicación con pacientes y colegas en estudiantes de sexto año de medicina que han recibido formación en comunicación desde primer año. Es un curso optativo de 5 semanas que se realiza parcialmente online, con sesiones presenciales semanales y una evaluación final. Como metodología para el aprendizaje y evaluación se utiliza la técnica de role play y se desarrolla un feedback grupal: del alumno involucrado, pares y docentes. En esta primera entrega se describe el curso, los temas troncales y la metodología de role play utilizada, además de una breve encuesta realizada al concluir. Se incluyen las condiciones fundamentales para dar feedback.

Como conclusión inicial, este curso aportaría a la formación integral de los estudiantes, al jerarquizar habilidades indispensables. Tanto el role play como el feedback grupal son altamente valorados por estudiantes y docentes.

Palabras clave: comunicación, feedback, profesionalismo, evaluación por pares.

\begin{abstract}
Communication skills are recognized as an essential competency in health professionals. Nowadays, it is important to develop them in order to instil professionalism in medicine students and graduates. In the present paper, we describe a course designed to optimize communication with patients and colleges, for medicine students on the sixth year of their University studies, who also received a related doctor-patient course during the first year. It is a five-weeks optional course, partially online and with five face-to-face sessions, and a final evaluation. The subjects and the role-play methodology used in the group sessions are described here. For the continuous and final evaluation, a multi-feedback is developed, based on the reflexive opinion from each student involved, the peers and faculty members. Essential conditions to give feedback are outlined and a brief survey at the end of our course is included.

As an initial conclusion, the course would contribute to the integral education of our students enhancing these essential skills. The role-play method as well as the multi-feedback are highly valued by students and faculty members.
\end{abstract}

Keywords: communication, feedback, professionalism, peer assessment.

Revista Methodo: Investigación Aplicada a las Ciencias Biológicas. Universidad Católica de Córdoba Jacinto Ríos 571 Bo Gral. Paz. X5004FXS. Córdoba. Argentina. Tel.: (54) 3514517299 / Correo: methodo@ucc.edu.ar / Web: methodo.ucc.edu.ar | ARTICULO ORIGINAL Rev. Methodo 2020;5(1):24- 


\section{Introducción}

La importancia de educar habilidades de comunicación en la formación de los médicos y estudiantes es ampliamente reconocida ${ }^{1,3}$. Constituyen un aspecto clave en el desarrollo de cada profesional de la salud y son posibles de educar, modelar y mejorar a lo largo de la vida. Se entiende la comunicación como una competencia, o sea la suma de conocimientos, habilidades y actitudes para superar desafíos complejos ${ }^{3}$. La bibliografía describe experiencias formativas en posgrado, pero son escasas en el grado, menos aún en planes de estudio, que como el nuestro incluye la RMP desde el primer año. Hoy se debería tender a que los estudiantes incorporen estas destrezas como parte fundamental del repertorio clínico, al internalizar desde tempranas etapas las conductas apropiadas que cimienten su identidad médica y los hagan comunicadores eficientes. En el marco de esa formación incluimos el feedback como herramienta para la incorporación y evaluación de habilidades ${ }^{5,12}$

\section{Antecedentes}

En 1997 la Facultad de Ciencias Médicas de la Universidad Nacional de Cuyo decidió realizar modificaciones trascendentes en su plan de estudios. Se buscó un cambio radical en la formación de los médicos, enfatizando en los aspectos humanos y sociales, se desarrollaron los contenidos en cursos integrados - no fragmentados en cátedras- y se instituyó el aprendizaje basado sobre problemas en reuniones tutoriales. Así también se implementaron los cursos de Relación Médico Paciente (RMP) siendo el del primer año de cursado anual, obligatorio y centrado en las habilidades de comunicación. (RMP1). Allí, cada estudiante se encuentra desde las primeras semanas de su carrera con pacientes diversos. Participando en pequeños grupos y supervisado por un tutor clínico, es iniciado en las destrezas para una buena comunicación.

En 2007 se realizó una evaluación del curso RMP1 a través de un extenso trabajo de tesis doctoral ${ }^{6}$. Como una parte del mismo 41 estudiantes del último año de la carrera realizaron entrevistas individuales filmadas con un paciente estandarizado (PE) que exponía una misma situación clínica. A posteriori, el total de alumnos fue dividido en grupos de 6 o 7 para participar en la sesión de evaluación de esas entrevistas filmadas. En cada sesión grupal estuvieron presentes dos docentes del Curso RMP1.

Tras observar cada uno de los videos, pares y docentes realizaron un feedback identificando libre y espontáneamente los aciertos y errores de cada estudiante en la entrevista evaluada. La sumatoria de las actitudes y conductas más apropiadas vertidas y comentadas por pares $\mathrm{y}$ docentes en el feedback grupal delineó el modelo de entrevista que en la opinión de ese grupo era más cercano a lo ideal.

Como una conclusión de esa experiencia, se destacó la alta valoración de los estudiantes y docentes sobre la práctica del feedback grupal, que veían como una herramienta clave para enseñar y evaluar habilidades de comunicación. Además, los estudiantes intervinientes coincidieron en la necesidad de profundizar y reimpulsar de una manera vivencial la formación en comunicación durante el ciclo clínico ${ }^{6,12,13}$.

Con esos antecedentes comenzamos en 2013 a desarrollar experimentalmente el curso para la PFO: "El encuentro con el paciente - habilidades de comunicación para los casi médicos", tras lograr la aprobación institucional correspondiente. Desde el año 2016 al 2019 han realizado el curso 217 estudiantes del sexto año, con incremento anual de inscriptos: de dos versiones anuales en 2016 con 16 inscriptos a 5 versiones en 2019 con 82 estudiantes, que libremente eligieron este curso optativo.

El objetivo de la primera parte del trabajo es la descripción del curso tal como se desarrolla actualmente en la carrera de medicina y la valoración inmediata del mismo por parte de los estudiantes.

\section{Metodología desarrollada}

El curso fue diseñado como un curso voluntario y optativo de cinco semanas, con las siguientes características, mantenidas en el tiempo:

-El grupo docente es multidisciplinario: clínicos, genetistas, psicólogos, pediatras, ginecólogos y oncólogos. El coordinador es médico clínico especialista en cuidados paliativos. El número de estudiantes por cursado varía entre 8 y 14 . Al principio se ofrecieron dos versiones anuales y por la demanda creciente en 2019 se repitió 5 veces al año.

-El cursado es semi-presencial: por una parte, se realizan tareas desde una plataforma virtual, donde se analiza semanalmente material relacionado con los temas troncales; los estudiantes realizan trabajos narrativos tras analizar videos, pinturas e historietas. Todo se discute diariamente por medio del chat y del servicio de mensajería.

-Por otra parte, una vez por semana se realiza una sesión grupal, donde se desarrollan cara a cara los

Revista Methodo: Investigación Aplicada a las Ciencias Biológicas. Universidad Católica de Córdoba. Jacinto Ríos 571 Bo Gral. Paz. X5004FXS. Córdoba. Argentina. Tel.: (54) 3514517299 / Correo: methodo@ucc.edu.ar / Web: methodo.ucc.edu.ar | ARTICULO ORIGINAL Rev. Methodo 2020;5(1):2429. 
temas reconocidos como clave. Estos son los siguientes:

-empatía y profesionalismo,

-entrevistas en diversas edades de la vida,

-abordaje de la sexualidad y la afectividad,

-entrevista motivacional,

- cómo dar malas noticias y

- la comunicación con colegas en el equipo de salud.

La metodología utilizada en las reuniones presenciales es la técnica del role play sobre situaciones clínicas habituales, donde un alumno juega el papel de médico y un par de docentes con otros alumnos, hacen de pacientes o familiares. Cada uno de los observadores atiende al desarrollo de la situación planteada y toma nota de todo lo sobresaliente positivo o negativo de la entrevista de modo espontáneo, sin que medie una escala o guía específica. Los aspectos que se valoran y corrigen son expuestos más adelante al comentar los resultados inmediatos.

Ejemplos de dos casos para role play:

1. A una guardia se presenta por tercera vez una mujer de 48 años disneica y ansiosa con su esposo - muy contrariado - y una hija. Están enojados porque no les solucionan el problema respiratorio, que se genera y agrava al discutir en casa. Ella recibe anticonceptivos orales y fue intervenida recientemente para reemplazo de cadera.

2.Una mujer de 39 años concurre feliz con su esposo, varón de 50 años, al médico de familia para control de embarazo dentro del primer trimestre y entregan al médico en sobre cerrado el estudio ecográfico. El mismo contiene el informe de un embarazo detenido, que la pareja desconocía.

Terminada la dramatización, las situaciones clínicas expuestas son valoradas y comentadas mediante un "multi feedback", que incluye: Auto evaluación, feedback de los pares y feedback docente.

1.Auto evaluación: consiste en una introspección del estudiante en rol de médico, en que reconoce y expresa tanto sus aciertos como sus deficiencias. También comenta sobre su percepción de los avances.

2.El feedback de los pares: es la devolución que hacen los pares sobre la misma teatralización, que en la bibliografía es destacado como tan preciso y efectivo como el feedback de los docentes. Esta devolución es especialmente valiosa, permite la apreciación llana y sincera de los compañeros que se involucran y a su vez aprenden ${ }^{4,11}$.

3.El feedback docente: cada uno analiza, señala aciertos o errores y termina dando su devolución reflexiva al estudiante, intentando optimizar el desempeño observado en todo el grupo.

\section{Modo de evaluación}

Durante el cursado, las actividades realizadas desde la plataforma virtual son leídas y comentadas por los docentes durante la semana, ofreciendo al estudiante la posibilidad de debatir y reflexionar acerca de los diversos tópicos. El concepto sobre la tarea realizada es integrado a la evaluación final del curso.

En las sesiones presenciales semanales se realiza una evaluación continua, a partir de observar la participación y la calidad de los role play que cada uno desempeña, así como sus aportes en el feedback de los compañeros.

El curso de 5 semanas culmina con un role play final para cada participante, tras lo cual el equipo docente coloca una calificación numérica a cada estudiante, valorando también su evolución a lo largo del curso.

En cada versión del curso ha aprobado la totalidad de los estudiantes que participaron. Cuando se observaron dificultades en la comunicación o inhibiciones, se orientó a cada alumno al área de asesoría pedagógica de la facultad, para diseñar estrategias de acompañamiento en esas áreas.

En la segunda parte de esta primera entrega abordaremos la valoración inmediata del curso por parte de los estudiantes.

\section{Evaluación inmediata de los estudiantes}

En una encuesta breve realizada en 2019 a 45 estudiantes al culminar el curso, se incluyeron tres preguntas para una valoración inicial de nuestra propuesta.

1-¿por qué buscó acrecentar su formación profesional en comunicación?

Las respuestas más frecuentes en orden decreciente de los estudiantes se sintetizan en las siguientes:

-Queda un buen recuerdo de lo aprendido en el curso RMP1

-En el resto de la carrera se enseña solo a "diagnosticar y tratar".

-Existe una carencia de vivencias de real comunicación con pacientes, más allá de la confección de la historia clínica tradicional.

-Los comentarios boca a boca entre compañeros del ciclo clínico en cuanto a que el curso era muy provechoso.

-No se han confrontado hasta ese momento con la posibilidad de reconocerse como una persona que construye su identidad médica y posee habilidades profesionales.

2. ¿qué se enfatiza en fomentar o corregir en el feedback de pares y docentes?

-Contacto visual y táctil

-Actitud empática

-Capacidad de escucha atenta - sostén de la mirada

Revista Methodo: Investigación Aplicada a las Ciencias Biológicas. Universidad Católica de Córdoba Jacinto Ríos 571 Bo Gral. Paz. X5004FXS. Córdoba. Argentina. Tel.: (54) 3514517299 / Correo: methodo@ucc.edu.ar / Web: methodo.ucc.edu.ar | ARTICULO ORIGINAL Rev. Methodo 2020;5(1):24- 
-Si interroga el modelo explicativo

-Tono de la voz

-Contenido de la comunicación y estructura de la entrevista

-Integración y aplicación de contenidos biomédicos incorporados en la carrera con la práctica de habilidades de comunicación

-Modo de informar y educar al paciente

-Capacidad para motivar cambios de hábitos

-Opciones terapéuticas centradas en el paciente

3. ¿Qué opina del feedback grupal?

En cuanto al feedback de los pares, los estudiantes aprecian positivamente ser evaluados por iguales. Valoran conocer sus aciertos y errores en forma directa, en un ambiente seguro y con pares que también están en la misma situación.

El feedback de los docentes fue también altamente apreciado y esperado por los estudiantes, en especial por la trasmisión de la experiencia desde las diversas perspectivas.

\section{Discusión}

Creemos que este curso cumple con una necesidad en la formación integral de los estudiantes al final de la carrera de grado, ya que jerarquiza habilidades indispensables, en una etapa crucial de la vida médica. Una buena capacidad de comunicación es una de las bases fundamentales del profesionalismo ${ }^{4,7}$.

Las ventajas de la metodología usada serían las siguientes:

-La preparación de las actividades presenciales a través de un trabajo previo en la plataforma virtual, que envía un mensaje uniforme, que es indispensable y motivador, a la vez pasible de ser evaluado.

-La integración de los aspectos biomédicos - en que los alumnos se sienten en parte competentes con la comunicación centrada en el paciente, comprendiendo una perspectiva más amplia: psicológica, familiar y social.

-La multiplicidad del feedback, que incluye autoevaluación y la opinión de pares y docentes. A diferencia del método tradicional de evaluación unidireccional, aquí predominan el diálogo y debate constructivos.

-La aceptación de las críticas conduce a una introspección sobre fortalezas y debilidades, para mejorar las competencias profesionales.

-Para aprobar se tiene en cuenta la participación, el compromiso con el feedback, la actitud empática, o lo atinado de una respuesta o reacción concreta, elementos subjetivos que en la tradición de la educación médica son raramente utilizados para enseñar y evaluar.

-El poder valorar comportamientos de un par con honestidad permite reflexionar sobre el propio actuar, para ejercitar y criticar las conductas no apropiadas.

-El curso cuenta con evaluación final, de modo tal que cada estudiante será aprobado y tendrá una calificación, según haya sido su trabajo online y el desempeño en las sesiones presenciales.

En cuanto a las debilidades, la subjetividad del feedback sería quizás, una desventaja, al no estar sujeta a una lista concreta de cotejo. Sin embargo, hoy esa subjetividad es vista como una fortaleza por diversos autores, cuando se suman las observaciones espontáneas de varios involucrados. En definitiva, lo que los estudiantes y docentes comentan o corrigen hace a la esencia de una comunicación efectiva, tal como se lee en las respuestas a la pregunta 2 de la encuesta inmediata. Muchos juicios subjetivos podrán dar una apreciación más confiable al final ${ }^{3}$.

El hecho que el accionar complejo y rico en aprendizajes de un estudiante deba ser transformado en una sola nota para adaptarlo al sistema evaluador sumatorio tradicional, es otra limitación difícil de superar por ahora.

El método del role play utilizado, sin recurrir a pacientes reales, es desde un punto de vista una desventaja. Sin embargo, la bibliografía otorga respaldo al método usado en este curso o el uso de pacientes estandarizados cuando se trata de estudiantes. Para la evaluación de profesionales en posgrado se recomienda la observación directa con pacientes reales en ámbitos clínicos auténticos ${ }^{3,9}$. Al ser la formación en estas habilidades un proceso educativo continuo y longitudinal a lo largo de la vida profesional, el curso deberá ser visto como un aporte parcial en ese camino.

En cuanto al feedback, ampliamente utilizado en nuestro curso, consideramos que es esencial para la educación y la evaluación de habilidades de comunicación. Es imposible construir una imagen de sí mismo sin la influencia del mundo exterior ${ }^{2}$. Muchos docentes en carreras de salud, conciben al feedback como una información unidireccional hacia el estudiante, tal como recordarle que debe adjuntar datos de laboratorio o que olvidó considerar otro diagnóstico diferencial. Tampoco es un examen. Creemos que ofrecer feedback no es una tarea sencilla en habilidades de comunicación, ya que se evalúan conductas complejas y cada evaluador se expresa desde su propia experiencia. Idealmente es una confrontación constructiva, fruto de un diálogo reflexivo con una persona con la cual se está vinculado, aliado. Y la calidad de

Revista Methodo: Investigación Aplicada a las Ciencias Biológicas. Universidad Católica de Córdoba. Jacinto Ríos 571 Bo Gral. Paz. X5004FXS. Córdoba. Argentina. Tel.: (54) 3514517299 / Correo: methodo@ucc.edu.ar / Web: methodo.ucc.edu.ar | ARTICULO ORIGINAL Rev. Methodo 2020;5(1):2429. 
esa relación influye en el aprendizaje o rechazo de lo vertido en el feedback ${ }^{3}$.

La credibilidad, la honestidad y el compromiso de quien da el feedback también son clave, así como el contexto de la comunicación y si se logra modelar con eficiencia la conducta del estudiante ${ }^{5}$ 11,12 .

Se requiere además de un ámbito apropiado y confiable, donde quien aprende sea reconocido como un miembro activo, perteneciente a un grupo que avanza hacia el crecimiento en estas destrezas. En definitiva, lo que se pretende con el feedback es lograr despertar una motivación en quien se forma para modelarse en adelante como un comunicador hábil y versátil ${ }^{3,12}$.

Cada estudiante o profesional va a ir conformando un estilo original de comunicación con pacientes y pares que deberá ser respetado en su esencia. Esta autonomía se garantizaría más cuando quienes valoran la conducta también son los pares ${ }^{12}$.

\section{Tres reflexiones hacia adelante}

Creemos que en la educación de grado se requiere la conformación de un cuerpo cada vez más numeroso de docentes entrenado para integrar los contenidos de este curso al aprendizaje cotidiano de habilidades clínicas para el diagnóstico y tratamiento. La bipolaridad actual de enseñar conocimientos biomédicos por un lado $\mathrm{y}$ comunicación empática centrada en el paciente por otro lado, debería ser superada a corto o mediano plazo.

Subsisten preguntas fundamentales como: ¿se alcanzaron los objetivos o resultados esperados? ¿El entrenamiento influencia su conducta? ¿Son nuestros egresados mejores comunicadores en su actual vida profesional? ¿Qué obstáculos encuentran en su labor para aplicar las destrezas adquiridas en comunicación? Para responderlas hemos emprendido la evaluación mediante encuesta a quienes cursaron y hoy son médicos, lo que describimos en la parte 2 de este trabajo.

Finalmente, desde las facultades no conocemos el nivel de profesionalismo ni cómo es la comunicación con pacientes y colegas en los ámbitos del sistema sanitario. Allí es donde se van a desplegar o malograr las habilidades incorporadas en las aulas. Por lo tanto, la formación de mentores entre los residentes y los responsables de centros de atención primaria, como también de enfermeros, es otra tarea indelegable de las Escuelas de medicina. Consideramos que el objetivo final del proceso educativo es la generación y multiplicación de espacios clínicos donde los valores del humanismo y la comunicación sean fomentados, discutidos y actualizados en forma permanente ${ }^{7}$.
De esta manera se daría respuesta a las expectativas que tienen la comunidad y cada paciente, respecto de las competencias de los profesionales que cuidan de su salud y calidad de vida.

\section{Agradecimientos}

Héctor Abaca, Laura Santolín, Romina Viadana, Laura Rodríguez, Eliana Gutiérrez, Ana Lucía Osorio y Lara Kraiselburd por su participación activa en el dictado. A la Dra. Lila Bujaldón por la revisión crítica.

\section{Bibliografía}

1. ASPEGREN K. (1999) Teaching and Learning communication skills in medicine- a review with quality grading of articles. Medical Teacher, Vol 21, No6.

2. BANDURA, A. (1991) Social Foundation of Thought and Action: A Social Cognitive Theory (Englewood Cliffs, NJ, Prentice-Hall)

3. C. van der VLEUTEN, et al. (2019), Assessment of communication skills, Patient Educ Couns., https://doi.org/ 10.1016/j.pec.2019.07.007 Contents lists available at Science Direct Patient Education and Counseling journal homepage: www.else vie $\mathrm{r} . \mathrm{com} /$ locate /pateducou

4. CUSIMANO, M.C.; TING, D.K.; KWONG, J.L.; VAN MELLE, E.; MACDONALD, S.E.; CLINE, C. (2019) Medical Students Learn Professionalism in Near-Peer Led, Discussion-Based Small Groups. Teaching and Learning Medicine. Vol.31 Nr.3 307 - 318 DOI 10.1007/s10459-012-9401-0

5. ENDE J. Feedback in Clinical Medical Education, (1983) JAMA -Vol.250, No 6

6. ESTEVES Pedro Eliseo, Habilidades de comunicación en la formación médica. Tesis doctoral inédita, (2007) Facultad de Ciencias Médicas UNCuyo.

7. HAFFERTY F. (2006) Measuring Professionalism: A Commentary. in Measuring Medical Professionalism, editor Stern ET, Oxford University Press

8. J.C.J.M. DE HAES, F.J. OORT \&R.L. HULSMAN (2005) "Summative Assessment of medical students' communication skills and professional attitudes through observation in clinical practice" Medical Teacher, Vol 27, No 7, 2005, pp 583-589. 
9. JACKSON V, BACK A. (2011) Teaching Communication Skills Using Role-Play: An Experience-Based Guide for Educators Journal of Palliative Medicine Volume 14, Number 6, Mary Ann Liebert, Inc. DOI: 10.1089/jpm.2010.0493

10. KLAMEN D., WILLIAMS R. (2006): Using Standardized Clinical Encounters to Assess Physician Communication, in Measuring Medical Professionalism, editor Stern ET, Oxford University Press

11. RAMM, D THOMSON A, JACKSON, A. (2015) Learning clinical skills in the simulation suite: the lived experiences of student nurses involved in peer teaching and peer assessment Nurse Education Today 35 823-827
12. TEN CATE TJ. (2013) Why receiving feedback collides with self determination. Adv in Health Sci Educ. 18:845-849

13. TEN CATE, TH, DE J.C.J.M, DE HAES (2000): Summative Assessment of medical students in the affective domain. Medical Teacher, Vol.22, No 1.

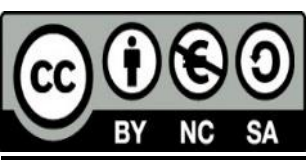

